\title{
Synthesis of Fluorine-containing Polyacrylamide Block Copolymer and Their Application for Rapidly Formation of Sub-10 nm Microdomains
}

\author{
Zhilong Li and Hai Deng* \\ Department of M acromolecular Science, State K ey Laboratory of M olecular Engineering of P olymers, \\ Fudan U niversity, Shanghai 200433, China \\ *haideng@ fudan.edu.cn
}

\begin{abstract}
Rapidly growing demand of shrinking the features of integrated circuits has accentuated the need for the next generation lithography. Among them, directed self-assembly (DSA) of block copolymers (BCPs) has attracted great interest due to their capability to form ordered nanopattern with high resolution and low cost. In this regard, several groups have investigated some high resolution DSA materials. However, all these materials required high annealing temperature or long annealing time. In this work, a series of polyacrylamide-based fluorine-containing BCPs were synthesized via reversible addition-fragmentation chain-transfer polymerization. One of the BCP materials can form sub-10 nm domains within $1 \mathrm{~min}$ at $80{ }^{\circ} \mathrm{C}$. A domain spacing of $\mathrm{BCP}$ with hexagonal structure was further confirmed by SEM on a silicon wafer.
\end{abstract}

Keywords: Block copolymer, Fast self-assembly, Sub-10 nm, Lithographic materials

\section{Introduction}

The semiconductor manufacturing has been conducted by Moore's Law for more than half century. The lithographic material plays the crucial role throughout the whole process. Recently, $13.5 \mathrm{~nm}$ extreme ultraviolet lithography (EUVL) has been deeply developed for $5 \mathrm{~nm}$ and $3 \mathrm{~nm}$ node, which is widely regarded as the potential candidate for next generation lithographic patterning technology $[1,2]$. However, due to its expensive cost and low throughput of EUVL, the industry still keeps searching another alternative patterning technology. The directed self-assembly (DSA) of BCPs has attracted ongoing interest due to its potential applications in sub-10 $\mathrm{nm}$ even sub-5 $\mathrm{nm}$ node lithographic patterning technology [3-5]. Poly(styrene-block-methyl methacrylate) (PS-b-PMMA) is one of the most classic BCPs for self-assembly [6,7]. However, due to its intrinsic relatively low $\chi$ (Flory-Huggins interaction parameter), the highest resolution is up to $22 \mathrm{~nm}$ pitch for a lamellar morphology, which significantly limits its application in lithographic process since sub-10 nm features is generally predominant in semiconductor industry insofar [8].

Recently, some high $\chi$ BCPs for sub-5 nm patterning has been extensively investigated by several research groups throughout the world [9-14]. A major drawback of these materials is relatively long thermal annealing time or high annealing temperature to form ordered nanostructure which is incompatible with the standard of manufacturing process.

Our previous studies have demonstrated that fluorine-containing block can accelerate self-assembly and lower down the annealing temperature [15-17]. Based on this unprecedented result, we designed and synthesized fluorine-containing polyacrylamide BCPs in this study (Fig. 1). By precisely controlling the property of two blocks and optimizing the annealing conditions, we finally achieved sub- 10 $\mathrm{nm}$ micro phase-separated structures with ultra-fast assembly speed. Different pitches and morphologies were obtained by adjusting the ratio and content of two blocks via controlling 
polymerization method. Furthermore, poly-N-benzylacrylamide (PNBAA)-based BCPs require high temperature $\left(160{ }^{\circ} \mathrm{C}\right)$ to achieve the thermodynamic equilibrium and finally self-assembly to form long range ordered structure. Intriguingly, the annealing time and temperature of poly-N-cyclohexylacrylamide (PNCHAA)-based BCPs were much milder than the previous case. The long range ordered structure can be readily observed at $80{ }^{\circ} \mathrm{C}$ within $1 \mathrm{~min}$. Noteworthy that the finest half pitch of these BCPs is less than $10 \mathrm{~nm}$, which meets the requirements of semiconductor patterning materials with small features.

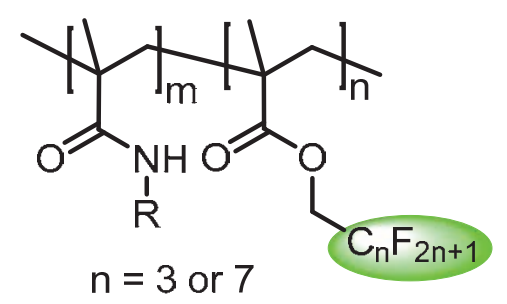

Fig. 1. General structures of fluorine-containing polyacrylamide BCPs.

\section{Experimental}

2.1. Synthesis and characterization

In this study, a series of BCPs with low polydispersity index (PDI) $(<1.15)$ were synthesized by reversible addition-fragmentation chain transfer (RAFT) polymerization. All the monomers, initiators were purchased from Energy Chemical; RAFT reagents were purchased from Strem. All the solvents (THF, hexane, toluene) were obtained from Titan. All the other reagents and solvents were used as received unless otherwise noted. After the polymerization, all the polymer was purified by dissolution and precipitation following with gel permeation chromatography (GPC) and nuclear magnetic resonance (NMR) analysis.

${ }^{1} \mathrm{H}-\mathrm{NMR}$ spectra were acquired on a $400 \mathrm{MHz}$ AVANCE III instrument using $\mathrm{CDCl}_{3}$ as solvent and TMS as an internal standard. From the ${ }^{1} \mathrm{H}-\mathrm{NMR}$ spectra, we can determine the characteristic peak of each component and calculate the molar ratio of two blocks. GPC measurements were used to calculate the molecular weight and PDI. Narrowly distributed polystyrene samples were used as calibration standards. All the BCPs show a narrow distribution up to 1.15 during the synthetic process. Small-angle X-ray scattering (SAXS) patterns were collected on a Nanostar SAXS (Bruker AXS GmbH, Germany), with pinhole collimation for point focus geometry.

\subsection{Thermal annealing for SAXS analysis}

As for the sample preparation for SAXS measurement, $5 \mathrm{wt} \%$ BCP solution was drop-cast on a silicon wafer and then dried in vacuum oven at room temperature for $2 \mathrm{~h}$. To guarantee the exact time of the thermal annealing process is 1 min, we chose the icy plate to chill the samples after annealing at corresponding temperature. After thermal annealing, we got the lamella or hexagonal structures with sub-10 nm half-pitch. Phase separation morphologies and micro domain spacings (d) of the BCPs were fully characterized by SAXS.

\subsection{DSC analysis}

DSC measurements were carried out on TA Q2000 and taken within the temperature range from $-40{ }^{\circ} \mathrm{C}$ to $160{ }^{\circ} \mathrm{C}$. Each thermal scan was taken at a rate of $20{ }^{\circ} \mathrm{C} / \mathrm{min}$ upon a second heating.

\subsection{SEM sample preparation}

The silicon templates were provided by Semiconductor Manufacturing International Corporation (SMIC). Sample preparation and thermal annealing process were shown in Fig. 2. The $5 \mathrm{wt} \%$ BCP solution was spin-coated on the silicon wafer at $3000 \mathrm{rpm}$ for $40 \mathrm{~s}$, and then baked at $160{ }^{\circ} \mathrm{C}$ for $1 \mathrm{hr}$.
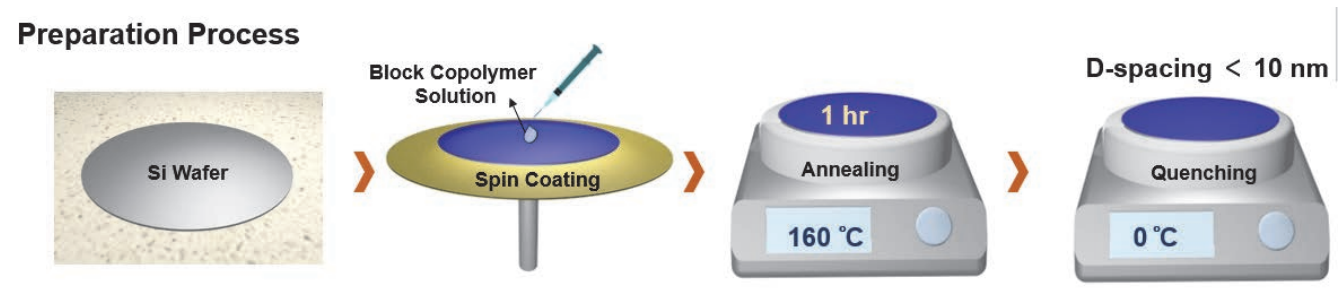

Fig. 2. Schematic diagram of the sample preparation and thermal annealing process for SEM analysis. 


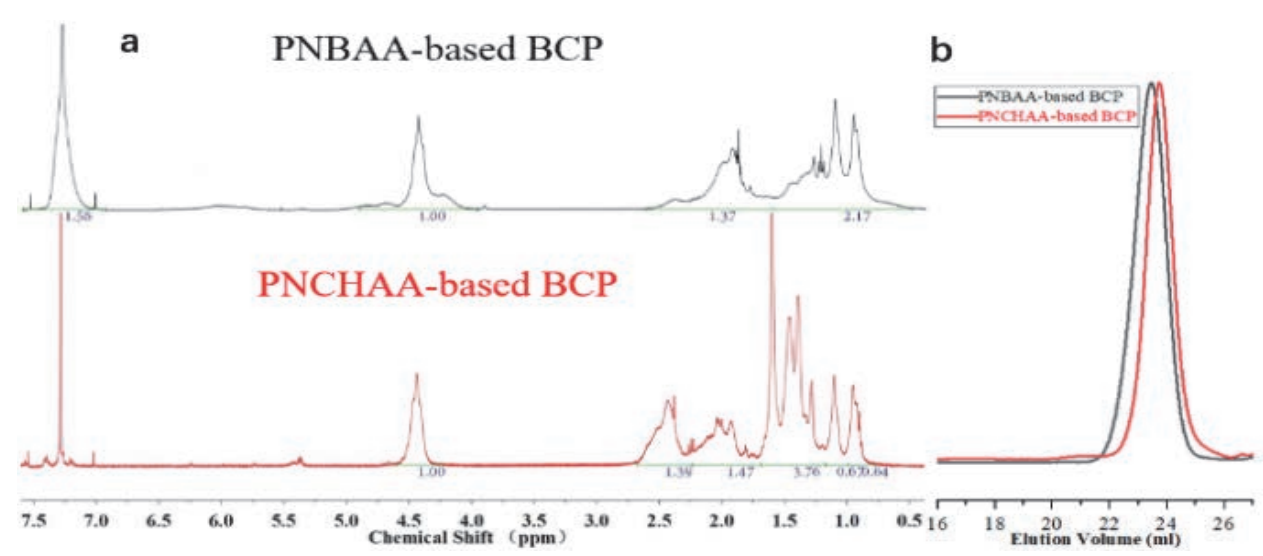

Fig. 3. (a) ${ }^{1} \mathrm{H}$ NMR spectra and (b) GPC curves of the samples.

\section{Results and discussion}

\section{1. ${ }^{1} \mathrm{H}-\mathrm{NMR}$ and GPC result of the samples}

The NMR spectrum exhibits that N-benzyl group of PNBAA-based BCP can be clearly observed (Fig. 3a). In contrast, the cyclohexyl peaks of PNCHAA-group was confirmed at $1.5-2.5 \mathrm{ppm}$.

The number of molecular weight $\left(\mathrm{M}_{\mathrm{n}}\right)$ were measured by GPC in THF with polystyrene standards. The $\mathrm{M}_{\mathrm{n}}$ of PNBAA and PNCHAA-based $\mathrm{BCP}$ is $10.5 \mathrm{k}$ and $8.2 \mathrm{k}$ respectively (Fig. 3b).

\subsection{SAXS results of PNBAA-based BCP}

Two independent polyacrylamide-based BCPs were synthesized by change the content and molar ratio of the monomers. By optimization of the annealing conditions, different phase separation pitches and morphologies were obtained. These block copolymers showed the potential as DSA material with high resolution for sub-10 $\mathrm{nm}$ lithographic patterning technology at low temperature. The domain spacing $\left(\mathrm{L}_{0}=\right.$ $\left.2 \pi / q^{*}\right)$ of BCPs is calculated by SAXS results, peaks with $q / q^{*}$ of $1,2,3,4$ indicating the forming of lamella (Lam) morphology. In contrast, peaks with $q / q^{*}$ of $1, \sqrt{3}, 2$ indicating the forming of hexagonal (Hex) morphology.

As shown in Fig. 4, the PNBAA-based BCPs, was inert to self-assemble at low temperature $(80$ ${ }^{\circ} \mathrm{C}$ ). When the hot plate was elevated to $160{ }^{\circ} \mathrm{C}$, rapid self-assembly was observed even in $1 \mathrm{~min}$ and no further distinctive change of morphology occurred after $1 \mathrm{~h}$. Hexagonal morphology was finally obtained with $12.5 \mathrm{~nm}$ at $160{ }^{\circ} \mathrm{C}$.

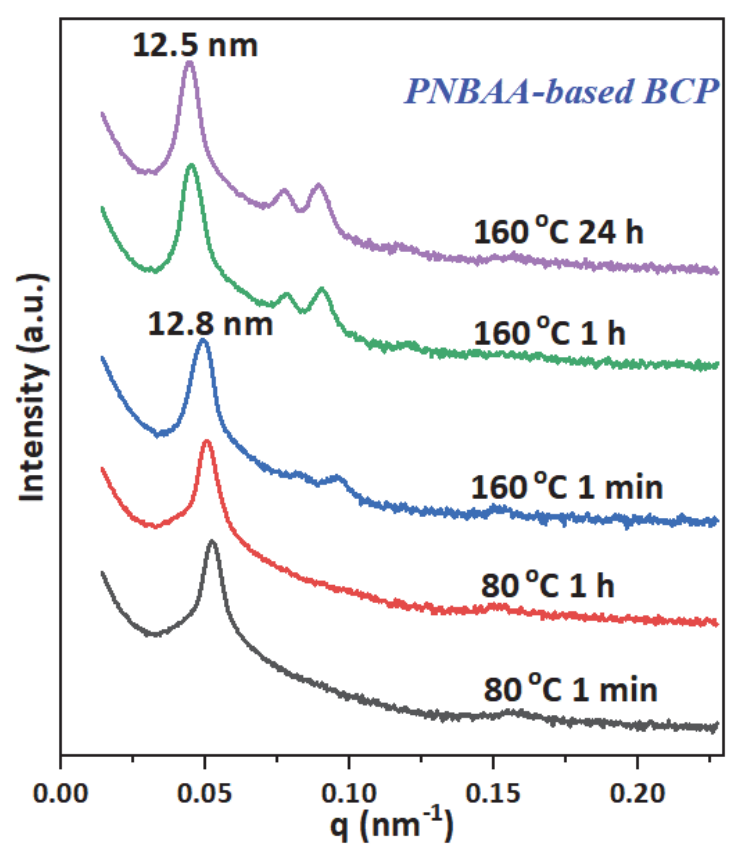

Fig. 4. SAXS profiles of PNBAA-based BCPs after thermal annealing at $80{ }^{\circ} \mathrm{C}$ for $1 \mathrm{~min}$ and $1 \mathrm{~h}$, at 160 ${ }^{\circ} \mathrm{C}$ for $1 \mathrm{~min}, 1 \mathrm{~h}$ and $24 \mathrm{~h}$, respectively.

\subsection{SAXS results of PNCHAA-based BCP}

When the more hydrophilic group was introduced into the system, the polymer can be directly phased separated at $80{ }^{\circ} \mathrm{C}$ within $1 \mathrm{~min}$ as shown in Fig. 5. The domain spacing of this PNCHAA-based BCPs is $12.3 \mathrm{~nm}$ with lamella morphology after $1 \mathrm{~min}$ annealing at $80{ }^{\circ} \mathrm{C}$. Compared with the PNBAA-based BCPs, PNCHAA-based BCPs need relatively shorter annealing time at much lower temperature. Interestingly, we also observed that the morphology might change to hexagonal when elevated the temperature to $160{ }^{\circ} \mathrm{C}$ in Fig. 5. 
However, the ordered structure also started to diminished when we prolonged the annealing time to $24 \mathrm{~h}$ presumably due to intrinsic decomposition of the polymer.

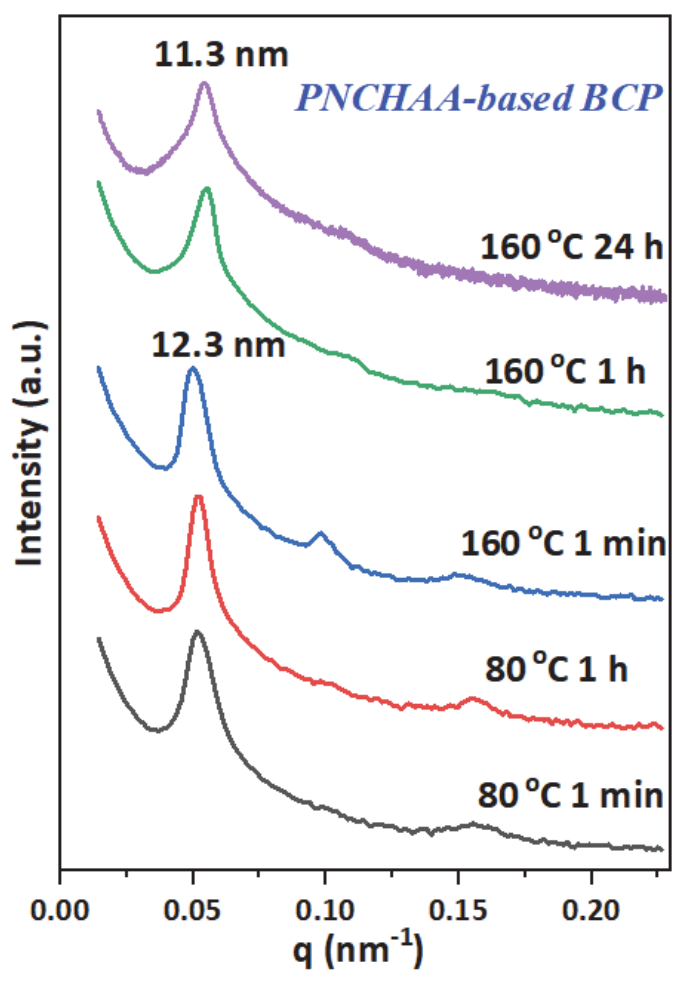

Fig. 5. SAXS profiles of PNCHAA-based BCPs after thermal annealing at $80{ }^{\circ} \mathrm{C}$ for $1 \mathrm{~min}$ and $1 \mathrm{~h}$, at 160 ${ }^{\circ} \mathrm{C}$ for $1 \mathrm{~min}, 1 \mathrm{~h}$ and $24 \mathrm{~h}$.

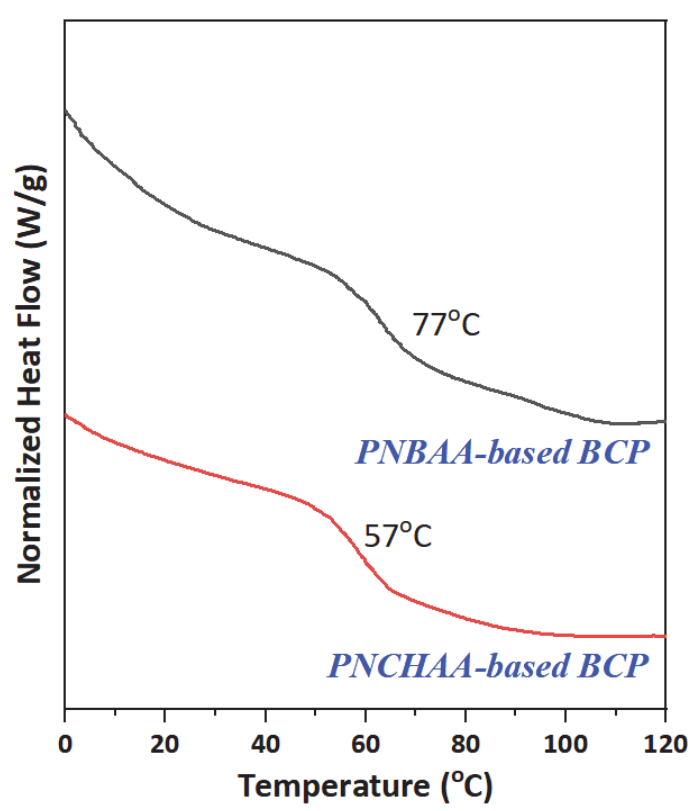

Fig. 6. DSC thermograms of two polyacrylamide based BCPs were labeled in the diagram.

\subsection{Single $T_{g}$ of BCPs}

The glass transition temperature $\left(\mathrm{T}_{\mathrm{g}}\right)$ was measured by DSC (Fig. 6). Only one $\mathrm{T}_{\mathrm{g}}$ was observed: $77{ }^{\circ} \mathrm{C}$ for PNBAA-based BCP and $57{ }^{\circ} \mathrm{C}$ for PNCHAA-based BCP.

\subsection{SEM results on silicon wafer}

As mentioned in our previous studies, these BCP series was unable to form the perpendicular lamellar morphology so we can't observe any nanopattern for PNCHAA-based BCPs in SEM. On the other hand, the hexagonal morphology of PNBAA-based BCP was successfully observed. After spin-coated on the template wafer and then dried in vacuum for $2 \mathrm{~h}$, the BCP thin film was annealed and self-assembled. In order to improve the contrast of image, samples for SEM measurement were post $\mathrm{CF}_{4}$ plasma etch for $45 \mathrm{~s}$ at $75 \mathrm{mTorr}$, and RF power is $10 \mathrm{~W}$. As displayed in the SEM image (Fig. 7), the hexagonal line pattern was formed on silicon wafer after thermal annealing at optimized conditions.

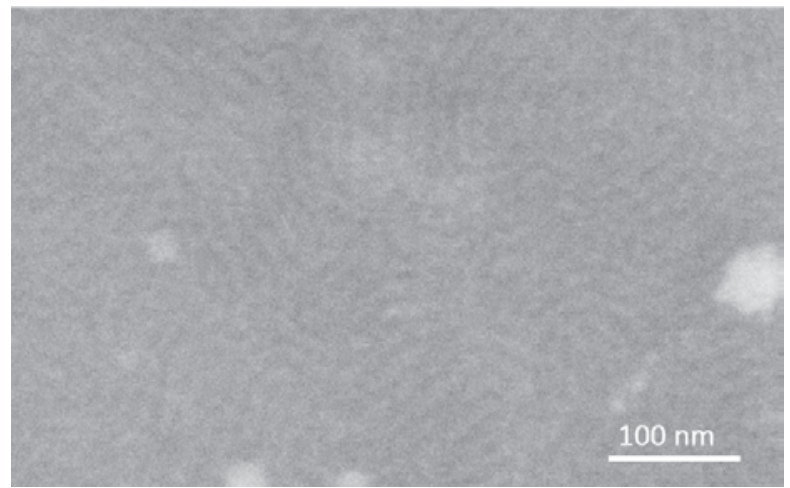

Fig. 7. FESEM image of PNBAA-based BCP (with hexagonal morphology) self-assembly on Silicon wafer at $160{ }^{\circ} \mathrm{C}$ for $1 \mathrm{~h}$.

\section{Conclusion}

In this study, we designed and synthesized a series of polyacrylamide-based BCPs. Based on our previous reported system, by tentative monomer design and precise control of both molecular weight and molar ratio of two blocks, we can eventually obtain the desired BCP with high resolution to $10 \mathrm{~nm}$. Additionally, PNCHAA-based BCPs was proved to form ordered nanostructures at ultra-fast assembling speed $\left(80{ }^{\circ} \mathrm{C}, 1 \mathrm{~min}\right)$. By introducing the fluorine-containing block, we can conveniently construct different nanostructure materials between 10 5 nm, which will be applied for DSA lithography as well as other functional nanomaterials. More detailed result and further 
investigation of pattern transfer is still undergoing in our laboratory and will be reported in due course.

\section{Acknowledgements}

This work was supported financially by Fudan University (IDH1717041), China Postdoctoral Science Foundation (KLH1615188), and Science and Technology Commission of Shanghai Municipality (No. 18511104900). The authors also acknowledge experimental support from the State Key Laboratory of Molecular Engineering of Polymers and the Nano-fabrication Laboratory of Fudan University.

\section{References}

1. B. Wu and A. Kumar, J. Vac. Sci. Technol. B, 25 (2007) 1743.

2. International Technology Roadmap for Semiconductors (ITRS), http://www.itrs.net/home.html, accessed: May, 2015.

3. H.-C. Kim, S.-M. Park, and W. D. Hinsberg, Chem. Rev., 110 (2010) 146.

4. D. J. C. Herr, J. M ater. Res., 26 (2011) 122.

5. L. Wan, R. Ruiz, H. Gao, K. C. Patel, T. R. Albrecht, and J. Yin, ACS Nano, 9 (2015) 7506.

6. H. Benoit, W. Wu, M. Benmouna, B. Moser, B. Bauer, and A. Lapp, Macromolecules, 18 (1985) 986.
7. J. Bang, S. H. Kim, E. Drockenmuller, M. J. Misner, T. P. Russell, and C. J. Hawker, J . Am. Chem. Soc., 128 (2006) 7622.

8. Y. Zhao, E. Sivaniah, and T. Hashimoto, Macromolecules, 41 (2008) 9948.

9. S. Park, D. H. Lee, J. Xu, B. Kim, S. W. Hong, U. Jeong, T. Xu, and T. P. Russell, Science, 323 (2009) 1030.

10. J. G. Kennemur, L. Yao, F. S. Bates, and M. A. Hillmyer, M acromolecules, 47 (2014) 1411.

11. D. P. Sweat, M. Kim, S. R. Larson, J. W. Choi, Y. Choo, C. O. Osuji, and P. Gopalan, Macromolecules, 47 (2014) 6687.

12. Y. D. Luo, D. Montarnal, S. Kim, W. C. Shi, K. P. Barteau, C. W. Pester, P. D. Hustad, M. D. Christianson, G. H. Fredrickson, E. J. Kramer, and C. J. Hawker, Macromolecules, 48 (2015) 3422.

13. A. P. Lane, X. M. Yang, M. J. Maher, G. Blachut, Y. Asano, Y. Someya, A. Mallavarapu, S. M. Sirard, C. J. Ellison, and C. G. Willson, ACS Nano, 11 (2017) 7656.

14. J. Kwak, A. K. Mishra, J. Lee, K. S. Lee, C. Choi, S. Maiti, M. Kim, and J. K. Kim, Macromolecules, 50 (2017) 6813.

15. X. Li, J. Li, and H. Deng, J. Photopolym. Sci. Technol., 30 (2017) 83.

16. X. Li, J. Li, C. Wang, Y. Liu, and H. Deng, J . Mater. Chem. C, 7 (2019) 2535.

17. C. Wang, X. Li, and H. Deng, ACS Macro Lett., 8 (2019) 368. 